\title{
Providing a Model for Creating Trust and Guaranteeing the Originality of Goods in the Machine Woven Carpet Supply Chain Based on Blockchain
}

\author{
Fatemeh Ghovanlooy Ghajar \\ Dept. Electrical and Computer \\ Engineering \\ National Technical University \\ of Athens \\ Athens, Greece
}

\author{
Javad Salimi Sartakhti \\ Dept. Electrical and Computer \\ Engineering \\ University of Kashan \\ Kashan,Iran
}

\author{
Ali Dorri \\ The School of Computer \\ Science and Engineering \\ UNSW, Sydney and DATA61 \\ CSIRO, Australia.
}

\begin{abstract}
For the past few years, globalization of collaborate industries and consumers has become an outstanding issue in the world. Collaboration between networks of all people/organizations, from creation to product sale as supply chain, is a hot challengingtopic. Planning and risk management, cost control, transparency and traceability are the challenges of supply chain. These challenges are very important topics as they are directly related to security and trust between members is doomed to failure.

The same problem appears in carpet industry supply chain. Lack of trust among network members and high potential of cheating in raw material and production process are serious problems in carpet supply chain. We suggest an infrastructure for carpet supply chain based on blockchain. Blockchain can provide security and reliability.Blockchain technology can be used as a decentralized information system which provide an information platform for all supply chain parts. Our proposed system can provide the traceability with trusted information in the entire carpet supply chain, whichwould effectively guarantee the carpet originality, by gathering, transferring and sharing theauthentic data of carpet in production, processing, warehousing, distribution, and selling. Our proposed model remove concerns and make a better commercial carpet market.
\end{abstract}

Keywords: Blockchain, Trust, Carpet Industry, SupplyChain,Traceability

\section{INTRODUCTION}

Machine carpet industry is one of the leading industries in Iran that has been able to incorporate traditional handmade carpets with state of the art technology. Carpet production involves a wide range of industries and suppliers. Paying attention to the quality of materials used, the basic characteristics and the need for transparency of information for those involved in this supply chain, need more attention in carpet supply chain management. Supply chain management can be considered as a process of planning, implementing and controlling related operations [1]. Supply chain is important in terms of production, storage and delivery [2].

The main goal of supply chain management is to reduce risks. Among the various risks that organizations face,communication risks such as participation of business colleagues in information distortion and fraud are very important $[3,4]$. The possibility of this business cooperation in distorting the information also is a very high risk in the industry. The consumers should be able to confirm the authenticity and originality of the materials used during the production of a particular product. Lack of transparency in supply chain process may lead to consumer dissatisfaction. [59]. In some supply chains such as food, RFID is used to build a supply chain tracking system [10].

In recent years, blockchain technology has received tremendous attention to serve as a secure transparent framework for supply chain management.Blockchainhas the potential to be integrated with other IoT technologies to ensure food safety [11]. Blockchain can have a significant impact on supply chain management. Some features of blockchain technology such as consistency and transparency for data storage; and intercept block's transactions for network members are very useful for supply chain [12]. One of the interesting benefits of using Blockchainin supply chain is to confirm the authenticity of manufactured goods.
Blockchain helps to maintain a healthy supply chain and reduce the risk of unhealthy and counterfeit goods [13]. Blockchain also allows irreversible transaction in the supply chain, and let the supply chain partners have access to transaction history $[14,15]$ and allows customers to track the source and follow product changes. This way, it potentially reduces the risk of activities [16]. This technology can play a determinant role in clarifying supply chain transactions [17].

In this article, for the first time, the machine woven carpet supply chain is discussed and some solutions are provided to solve the problem of product originality andthe trust between the manufacturer and the consumer.

With the introduction of industrial machinery into the art of handmade carpets, a lot of changes were made in it, and the prerequisite of carpet production also moved toward industrialization. Creation of different carpets with a vast diversity of color, reed and density provides a wide range for manufacturers and users of carpet machines. Today, carpet industry is one of the important pillars of the Iranian economy and should be seriously considered. This industry creates a variety of jobs ranging from designing pattern to providing yarn and selling carpet. But given the lack of transparency in the production process and the problems such as cheating on the yarn, fraud in the presentation of the actual density and reeds of the carpet, have led to uncertainty about the originality of the product.

The presence of broker in the sale of carpet causes a significant difference in the price of carpet for the buyer than for the producer. This factor is at the expense of the domestic manufacturer, i.e. low profits for manufacturer and high prices for consumer. The issue of uncertainty is a big problem in the carpet industry and supply chain. Therefore, the main issue is to create trust about the originality and the price of the goods. 
Using blockchain in the supply chain, we want to solve the problem of trust and fraud, and propose a model based on Blockchain.

Therefore, no one can change the data recorded in Blockchain. Due to its high security and reliability, Blockchain has recently been widely studied and applied. As shown in Fig. 1, blockchain is a sequence of blocks. Each block holds a number of transactions. Transactions are created by the parties of a transaction and broadcast on the network. Each block is chained to its previous block by the hash of the previous block values. Therefore, any change in a particular block will result in the loss of integrity in the blockchain and will be identified and deleted by other members of the network. Additionally, given the nonce positioned in the block and solving the complex mathematical puzzles by the miner, the mined block is being broadcasted by the miner on the network.

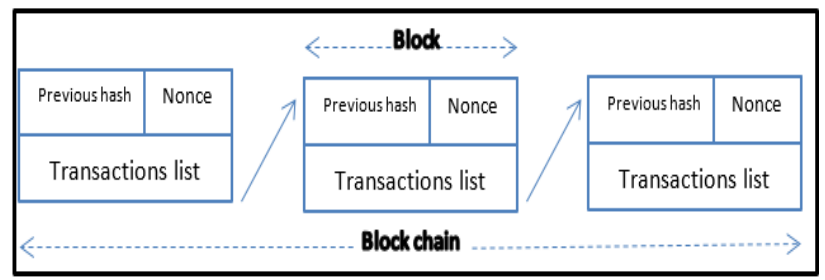

Figure 1-blockchain structure

Several methods have been proposed to choose a Miner for formation of block on Blockchain network. The most famous ones are proof of work, proof of stake, and proof of capacity [19], given the computational power, capital and storage capacity, which increases the chance of winning miners. Therefore, Blockchain provides a practical way to maintain data security, compatibility and reliability in the network.

The rest of the paper is organized as follow: In section 2 we explain the required material to proposing our Model. We will discuss the proposed model in section 3 . It presents an infrastructure for carpet supply chain based on blockchain technology. With attention to this model, we have a tracking system for members. This kind of system reduce risks for untrusted transaction between members. Due to using inputoutput transaction method, validation becomes easier and information transparency increases in the carpet supply chain. Evaluation ofthe proposed model is discussed in Section 4 while Section 5 provide conclusion and future work.

\section{BACKGROUND}

General issues in supply chain is followed by discussion on blockchain-based supply chain. First, we describe general issue and then we discuss supply chain, blockchain. Finally, we mention the blockchain usability for the traceability program of carpet supply chain.

With the fast growth of IoT, many researchers have focused on traceability of supply chain. Some of them pointed to the efficiency of traceability system by track ability of each product. In this case users can monitor anything from primary production to final disposal by consumer [20]. Some studies suggest a RFID based framework for food supply chain [21]. Using RFID for identification of product and biometric identifiers for the animal's identity in food supply chain, this system proposes traceability records.

Today, blockchain brings new approaches in many domains. Some of the research papers discussblockchain'spotential in manufacturing supply chain [22]. So it offers a good platform for distribution and transparent mechanism to be used in business and industry. Because of the lack of trust in business processes, some researchers offer blockchain for emerging distributed technology with data sharing for untrusted participants [23].

\subsection{Supply chain}

In manufacturing and business, supply chain is a management system of people, resources and activities involved from supplier stage to the consumer. And supply chain management is the management of product flowfrom beginning to the end. It involves business activity to maximize customer value and gain the advantage in market for producer. Supply chain management has two sub tasks [24]:

1) Activity planning, implementing and controlling to create a value for final consumer.

2) Integration and cooperation between companies for business process.

The important issue in the supply chain management is information and communication technology [25, 26]. Technology represents a good tool for supply chain innovation [27].

Table 1- Supply chain applications across industries [28].

\begin{tabular}{|c|c|}
\hline Industry & Count \\
\hline Food products & $9(31 \%)$ \\
\hline Container shipping & $7(24 \%)$ \\
\hline $\begin{array}{c}\text { Discrete } \\
\text { manufacturing }\end{array}$ & $4(14 \%)$ \\
\hline Pharmaceuticals & $3(10 \%)$ \\
\hline Mining industry & $2(7 \%)$ \\
\hline Other/not specified & 4 \\
\hline
\end{tabular}

\subsection{Blockchain}

Blockchain is used as a platform in decentralized networks to maintain consistent databases and build security and trust among all network members. Its first application was by Satoshi Nakamoto in creating Bitcoin as a cryptocurrency [18]. The difference between networks based on blockchain with centralized networks is in the absence of a central node.

All network members in Blockchain have equal positions, and they all keep a copy of the database (i.e., Ledger). So blockchain is a distributed ledger that is shared between peerto-peer (P2P) network's node [29, 30].

Research studies about blockchain application in supply chain focus on construction and integration supply chain through the blockchain [37, 38]. Using blockchain is very useful in transparency and traceability $[31,36]$. It enables supply chain for significant research advances [34] and helps for supply chain provenance and knowledge of product provenance [33, 35]. Also blockchain can be used for digital advertisingsupply 
chain, increasing resilience uncertainty and etc. [32]. In carpet supply chain, no study has focusedon this issue, so this work is novel and the first in this area.

\section{PROPOSED MODEL.}

\subsection{Main idea}

In this study, we are looking to build trust in the carpet machinery supply chain. In this industry, in addition to carpet manufacturers, raw material manufacturers, including yarn manufacturers, knitting manufacturers, and also manufacturers of accessories such as resin, leather, starch, and carpet packing covers are present in this supply chain to complete carpet production.

In Fig. 2, the supply chain diagram can be seen. This supply chain can include the following general steps:

$\begin{array}{ll}1- & \text { Supply of raw materials } \\ 2- & \text { Production } \\ 3- & \text { Processing } \\ \text { 4- } & \text { Warehouse } \\ \text { 5- } & \text { Sales. }\end{array}$
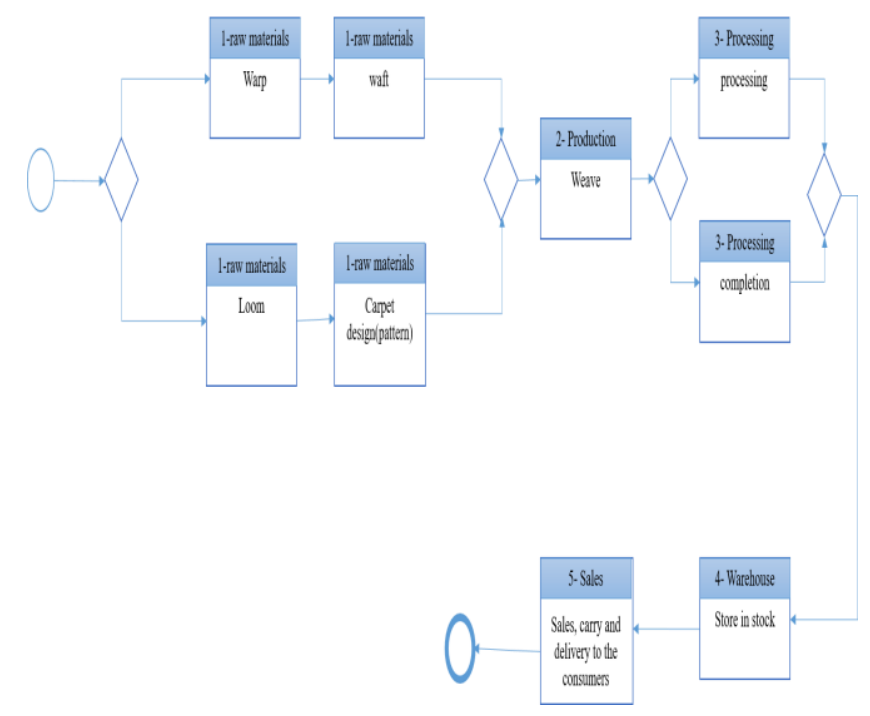

Figure 2: diagram of machine carpet

In the material supply phase, according to the characteristics of the carpet, the appropriate pile yarn, warp yarn and weft yarn should be provided. One of the most important concerns of end users is the authenticity of the warp yarn and weft yarn. At raw materials stage, the production transparency is achieved by registering all the characteristics of material, including the location of production and destination, productiontime and sales date, and qualities of yarn in a block in Blockchain. Based on yarn barcode information that exists on the block, every carpet producer can check yarn quality with this information, if raw material and block information match, then producer proves the block validity by referring block number on his own block.
In the production stage, the device and the carpet pattern information, including the type of pattern, layout and general information, the carpet texture information such as density, reeds, height of pile yarn, and carpet size can be fully recorded in a block for daily products. As the previous step, next chain player will check for authorization of the product. At the processing and completion stage, there is also the possibility of cheating on materials used, including resin, starch, type of leather and root type used.

At the processing and completion stage, there is also the possibility of cheating on materials used by manufacturer, including resin, starch, and leather and fringe type used. By registering the specification and percentage of material for each carpet in Blockchain, any fraud in the processing will be prevented. The production information of carpets, that is available on the network, is collected and placed in the blocks by the miners. The miners store new block in the blockchain following a consensus algorithm which ensures blockchain security(Please see Fig.3).

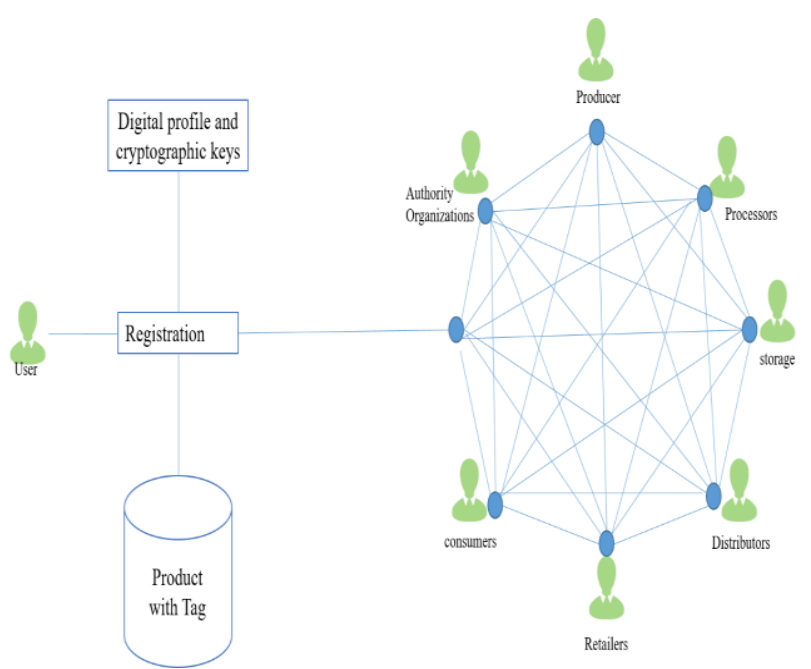

Figure 3- The Conceptual Framework of the proposed model and the nodes of the Blockchain Network

In the warehouse, the block address, which includes the transaction containing the carpet profile, is attached to the product as a barcode tag which will be visible to the buyers and thus insures transparency in supply chain. The fifth stage, the distribution and sale stage, can be done in two different ways; sell by retailers or direct sales from the factory.

\subsection{Proposed Blockchain Structure}

In the proposed blockchain-based system, transactions contain input-output, where the input is data of material block and output is production information.In each stage the producer 
generates a transaction, as shown in Figure 4, and broadcasts to the network.

Raw material block number
Verify block flag status
Producer public key (ID)
Puyer public key (ID)
Peduce time data (optional)

Figure 4-Transaction format.

In this way, the interests of the manufacturer and the buyer are directly supported. By providing a store on the blockchain platform, the manufacturer can directly sell the product without the presence of broker, and consumers can also buy the product at the best price. With this method, using smart contracts.

Each member of supply chainstorestheir product information in the transaction. This information contains raw materials, production time, producer name (public key), buyer name (public key) and metadatawhere applicable. In this method each member of supply chain must verifymaterial or product and mention their agreements on their transaction. The transactions are verified and stored in the blockchain by miners. Each node, focusing on their stack, can work as a miner and try to generate a block for exiting transactions. The stack definesthe trust value of the node in network. Each node can generate a trustable block and get $51 \%$ of network's nodes agreement, then the block linked to the main chain and miner's stack will be raised.

Each candidate miner must put a specified stake for his process, if the generated block is accepted by the network, they can get reward stake; otherwise they lose those amount of stake. However the miner incentive for participation in this competition is priority of higher stake owner to their generated transaction in the network by other miners.

To maintain the privacy of nodes, we suggest apermissioned blockchain. In the permissioned blockchainsall parties require permission to read the information on the blockchain, sowe can set who can transact and who can mine new blocks and add it into the chain. At the end, the information of final product issaved in RFID tag for reference to the block.

The first method is the traditional way of selling in stores and retailers, which is a common technique. The second approach, which we offer, is the use of smart contracts. This method can be seen in Fig. 5.

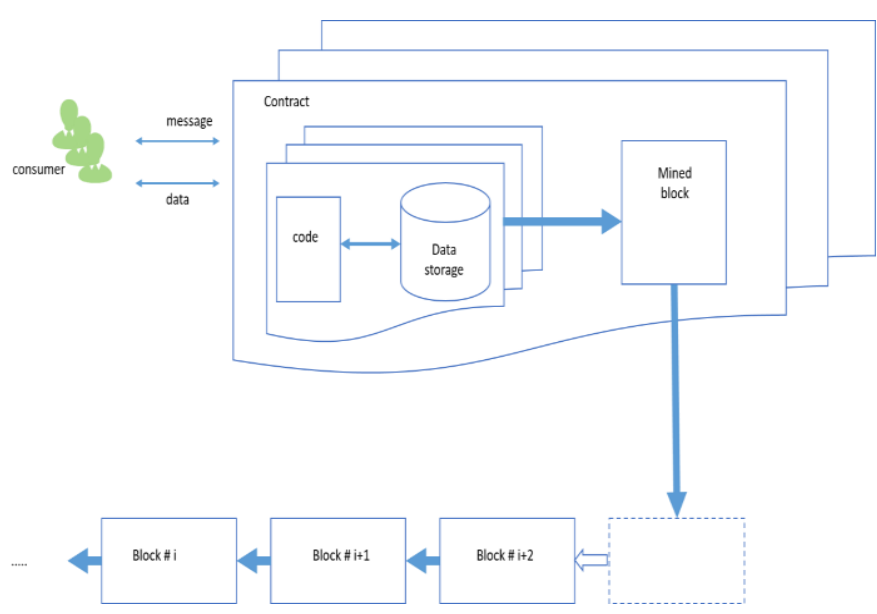

Figure 5-Sales Infrastructure with Block Chain Based on smart Contract.

the originality of the goods is guaranteed by the manufacturer and the payment of the price is also guaranteed by the consumer. All trading conditions are recorded in the smart contract. The cost is blocked from the buyer's account but will not be paid to the seller until the delivery of the goods. If the provisions are properly implemented, the transaction will be completed by the parties recorded in Block Chain. This will ensure full trust between the parties of this network. The blockchain process can be seen in Fig. 6 .

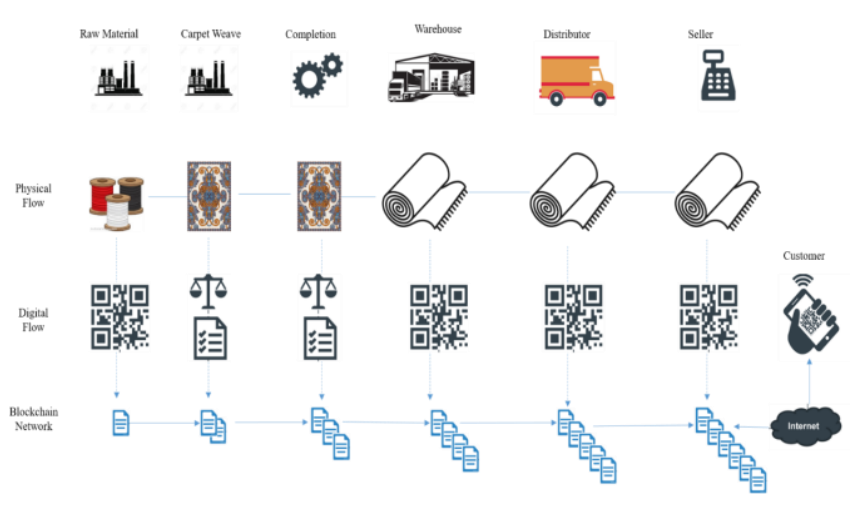

Figure 6- Machine carpet Supply chain based on blockchain

\section{DISCUSSION}

A blockchain platform enables tracking system in the supply chain. In this system, all transaction information can be used by a unique product ID. This ability is used for data collection in IoT (i.e., digital Flow). Blockchain has the same benefit for this system. Firstly blockchain's nature is distributed and compatible for distributed database in IoT layer. Secondly, transactions are accessible for every new node in our supply chain network. And finally due to using blockchain infrastructure, adding new nodes in supply chain is easy and ready for tracking system.

Transparency in supply chain creates trust, balances the information between suppliers and makes incentive to have good behavior on system. Besides, it makesconsumers sure 
that they have bought the original product and it's like a guarantee for every product.

Another benefit of this system is identification of malicious agents in the supply chain. The previous block is checked for using raw material to produce new product, and the stake of miners is checked to generate trustable block. This encourages the blockchain nodes to select the best way and have good behavior. So, with this solution, a malicious node is identifiedvery fast and all nodes will choose the way without cheating in the products.

\section{CONCLUSION}

In this article, we addressed the problems of the carpet supply chain, and found out that the possibility of fraud in the production process as well as the sale of this product is very high. The problem of collusion of raw material suppliers with the supply of false materials to carpet completion and the supply of misinformation at the time of sale is one of the main problems of this supply chain, which issolved

Many consumers can block chain technology in this industry, and we can guarantee the authenticity of the product and information of each carpet. Implementing this method, trust will be created between the producer and the consumer and the possibility of fraud will be very low.

\section{REFERENCES}

[1] Al-Jaroodi, Jameela, and Nader Mohamed. "Blockchain in Industries: A Survey." IEEE Access 7 (2019): 36500-36515.

[2] Kim, Hongyeun, et al. "Elastic properties of long periodic stacking ordered phases in Mg-Gd-Al alloys: A firstprinciples study." Intermetallics 98 (2018): 18-27.

[3] Baird, Inga S., and Howard Thomas. "What is risk anyway? Using and measuring risk in strategic management." Risk, strategy, and management 5 (1990): 21-54.

[4] Bettis, Richard A., and Vijay Mahajan. "Risk/return performance of diversified firms." Management Science 31.7 (1985): 785-799.

[5] Antony, Solomon, Zhangxi Lin, and Bo Xu. "Determinants of escrow service adoption in consumer-toconsumer online auction market: an experimental study." Decision Support Systems 42.3 (2006): 1889-1900.

[6] Featherman, Mauricio S., and Paul A. Pavlou. "Predicting e-services adoption: a perceived risk facets perspective." International journal of human-computer studies 59.4 (2003): 451-474.

[7] Kim, Dan J., Donald L. Ferrin, and H. Raghav Rao. "A trust-based consumer decision-making model in electronic commerce: The role of trust, perceived risk, and their antecedents." Decision support systems 44.2 (2008): 544-564.

[8] Mitchell, Vincent-Wayne. "Consumer perceived risk: conceptualisations and models." European Journal of marketing 33.1/2 (1999): 163-195.
[9] Sweeney, Julian C., Geoffrey N. Soutar, and Lester W. Johnson. "The role of perceived risk in the quality-value relationship: a study in a retail environment." Journal of retailing 75.1 (1999): 77-105.

[10] Tian, Feng. "An agri-food supply chain traceability system for China based on RFID \& blockchain technology." 2016 13th international conference on service systems and service management (ICSSSM). IEEE, 2016.

[11] Tian, Feng. "A supply chain traceability system for food safety based on HACCP, blockchain \& Internet of things." 2017 International Conference on Service Systems and Service Management. IEEE, 2017.

[12] Abeyratne, Saveen A., and Radmehr P. Monfared. "Blockchain ready manufacturing supply chain using distributed ledger." (2016).

[13] Holland, Martin, et al. "Copyright protection in additive manufacturing with blockchain approach." Transdisciplinary Engineering: A Paradigm Shift 5 (2017): 914-921.

[14] Abeyratne, Saveen A., and Radmehr P. Monfared. "Blockchain ready manufacturing supply chain using distributed ledger." (2016).

[15] Alzahrani, Naif, and NirupamaBulusu. "Block-supply chain: A new anti-counterfeiting supply chain using NFC and blockchain." Proceedings of the 1st Workshop on Cryptocurrencies and Blockchains for Distributed Systems. ACM, 2018.

[16] Yeung, Ruth, and Wallace MS Yee. "Food safety concern: Incorporating marketing strategies into consumer risk coping framework." British Food Journal 114.1 (2012): 40-53.

[17] Min, Hokey. "Blockchain technology for enhancing supply chain resilience." Business Horizons 62.1 (2019): 3545.

[18] Nakamoto, Satoshi. "Bitcoin: A peer-to-peer electronic cash system." (2008).

[19] Sankar, Lakshmi Siva, M. Sindhu, and M. Sethumadhavan. "Survey of consensus protocols on blockchain applications." 2017 4th International Conference on Advanced Computing and Communication Systems (ICACCS). IEEE, 2017.

[20] Folinas, Dimitris, IoannisManikas, and Basil Manos. "Traceability data management for food chains." British Food Journal 108.8 (2006): 622-633.

[21] Shanahan, C., et al. "A framework for beef traceability from farm to slaughter using global standards: an Irish perspective." Computers and electronics in agriculture 66.1 (2009): 62-69. 
[22] Abeyratne, Saveen A., and Radmehr P. Monfared. "Blockchain ready manufacturing supply chain using distributed ledger." (2016).

[23] Weber, Ingo, et al. "Untrusted business process monitoring and execution using blockchain." International Conference on Business Process Management. Springer, Cham, 2016.

[24] Council of Supply Chain Management Professionals. "Supply chain management terms and glossary." (2013).

[25] Gunasekaran, Angappa, and Eric WT Ngai. "Information systems in supply chain integration and management." European journal of operational research 159.2 (2004): 269295.

[26] Stadtler, Hartmut. "Supply chain management and advanced planning-basics, overview and challenges." European journal of operational research 163.3 (2005): 575588.

[27] Arlbjørn, Jan Stentoft, Henning de Haas, and Kristin BalslevMunksgaard. "Exploring supply chain innovation." Logistics research 3.1 (2011): 3-18.

[28] Blossey, Gregor, JannickEisenhardt, and Gerd Hahn. "Blockchain Technology in Supply Chain Management: An Application Perspective." Proceedings of the 52nd Hawaii International Conference on System Sciences. 2019.

[29] Crosby, Michael, et al. "Blockchain technology: Beyond bitcoin." Applied Innovation 2.6-10 (2016): 71.

[30] Sternberg, Henrik, and Giulia Baruffaldi. "Chains in Chains-Logic and Challenges of Blockchains in Supply Chains." Proceedings of the 51st Annual Hawaii International Conference on System Sciences. 2018.
[31] Kamble, Sachin S., AngappaGunasekaran, and Rohit Sharma. "Modeling the blockchain enabled traceability in agriculture supply chain." International Journal of Information Management (2019).

[32] Chen, Wubing, et al. "A Survey of Blockchain Applications in Different Domains." Proceedings of the 2018 International Conference on Blockchain Technology and Application. ACM, 2018.

[33] Kim, Henry M., and Marek Laskowski. "Toward an ontology-driven blockchain design for supply-chain provenance." Intelligent Systems in Accounting, Finance and Management25.1 (2018): 18-27.

[34] Jabbari, Arman, and Philip Kaminsky. "Blockchain and Supply Chain Management." (2018).

[35] Montecchi, Matteo, Kirk Plangger, and Michael Etter. "It's real, trust me! Establishing supply chain provenance using blockchain." Business Horizons (2019).

[36] Eljazzar, Maged M., et al. "Merging supply chain and blockchain technologies." arXiv preprint arXiv:1804.04149(2018).

[37] Rodrigo, M. N. N., et al. "Blockchain for construction supply chains: a literature synthesis." Proceedings of the 11th International Cost Engineering Council (ICEC) World Congress \& the 22nd Annual Pacific Association of Quantity Surveyors Conference, 18-20 November 2018, Sydney, Australia. 2018.

[38] Korpela, Kari, JukkaHallikas, and Tomi Dahlberg. "Digital supply chain transformation toward blockchain integration." proceedings of the 50th Hawaii international conference on system sciences, 2017. 